\title{
Analysis of Direction on Product Design in the Era of the Internet of Things
}

\author{
Baozhen Tian ${ }^{1, \mathrm{a}}$, Suihuai $\mathrm{Yu}^{1}$,Jianjie $\mathrm{Chu}^{1}$ and Wenhua $\mathrm{Li}^{1}$ \\ ${ }^{1}$ Shaanxi Engineering Laboratory for Industrial Design, Northwestern Polytechnical University, Xi'an,710072, China
}

\begin{abstract}
Internet of things (IoT) as a new revolution in science and technology will bring new vitality to the design. IoT has become the third revolution of the world industry information development after the world experienced the computer and the Internet. With the development of industry information, IoT as Internet service and applications expand through intellisense, identification technology, pervasive computing and ubiquitous network convergence applications. IoT era is the era of intelligent products. This article elaborates on the aspects of design content, design objects, design procedures and methods, means of obtaining design requirements, design tools, and changes in design direction. In the era of IoT, industry designers should focus on the interaction between humans and smart products and the interaction between smart products.
\end{abstract}

\section{Introduction}

The Internet of Things (IoT) has become a hot topic. Some applications in IoT are exploiting in healthcare, transportation, automotive industries, \& electronic meters and so on[1]. "IoT is an emerging network superstructure that connects physical resources and people together with software"[2]. Like any information system, the IoT will rely on a group of hardware, software and architectures. The basic idea of IoT is to connect all things in the world to the internet [3]. Through the IoT, different types of goods, sensors, and facilities can communicate and form pervasive networks that convenient our daily lives [4]. What the IoT represents is an evolution of the application of these existing technologies according to several of facilities as well as the interconnection of networks of these devices across the Internet [5]. Although there is no general definition for the IoT, the core concept is that everyday objects can be equipped with identifying, sensing, networking and processing capabilities that will allow them to communicate with each other and with other devices and services over the internet to achieve some useful objective [6]. With the help of intelligent and reliable, IOT connected things wirelessly. Objects with this facility will be able to communicate without any human interference[78].The IoT consists of objects, sensor devices, communication infrastructure, computational and processing unit that may be placed on cloud, decision making and action invoking system[9].As a new scientific and technological revolution, it will bring new vitality to the design.

According to predictions of relevant experts, the future trend is the globalization of information resources, and global management will rely on various information resources [10-11]. Iot will control these information network resources. With the developments in the internet technologies, technologies in sensor networks and near field communication using RFID (radio frequency identification devices) tags have also been evolving [12]. Therefore, some developed countries (such as the United States, the European Union, Japan, etc.), enterprises and academic groups have invested human resources, material resources, and financial resources to conduct indepth research on the IoT and have sought to make breakthroughs in this area.

Industrial design is bound to undergo profound changes as an important link closely connecting science and technology in the era of IoT. With the emergence of the IoT, it is a daunting task how to use the IoT technology better for design service, is suspends in front of the current industrial designer. It is not just combination the technology of IoT with the aesthetic knowledge simply, which need to test the ability and wisdom of a designer. Design as an initial impulse of humanity is the most fundamental human wisdom. This article puts forward some suggestions on issues that should be noted in the industrial design of IoT.

\section{Product design in the era of the internet of things}

"Intelligent type" is the characteristic of the IoT. How to design the intelligent products is worthy of further study to the industrial design researchers. The IoT is a paradigm where everyday objects can be configured with identifying, sensing, net-working and processing abilities that will allow them to interact with each other and with other facilities and services over the Internet to finish

\footnotetext{
" Corresponding author: ${ }^{a}$ tbz246@ mail.nwpu.edu.cn,
} 
some functions. The core concepts underlying the IoT are not new. For years, technologies such as RFID

( radio frequency identification devices) and sensor networks have been applied in industrial and manufacturing contexts for tracking large ticket items such as cranes and livestock. The IoT can find its utilizations in almost every aspect of our daily life such as: prediction of natural disasters; industry applications; water scarcity monitoring; design of smart homes; medical applications; agriculture application; intelligent transport system design; design of smart cities; smart metering and monitoring and smart security[13]. The literature [5] showed that "Intelligent" products can understand people's intentions and make judgments, to reason, to execute instructions issued by humans and to make proper handling of items in a certain extent. Intelligence is based on artificial intelligence, and given by designers and engineers. It is an intelligent and internet-linked product that belongs to a higher level of smart products. In the environment of IoT, the term "intelligent item" is often used. In addition, the smart item can be a single product, such as a smart book, or it can be a system composed of a number of products, such as a smart public transportation system, smart security facilities, and so on. Future lives should be leaded by designing, which requires the simplest way to achieve the ultimate goal. The scope of research required to achieve IoT at the scale envisioned future customers' needs requires significant research along many directions.

\subsection{Changes of design content}

Traditional design content includes functions, structures, forms, surface treatments, materials, colors, etc. Tremendous development will be acquired based on the content in the future of the IoT. In functionality, they will be greatly improved with the development of IoT technology. The complexity of the function will complicate the design. In the structure, the rapid prototyping equipment interacts with the computer intelligently to realize the design of analog structures and to complete the production process of the structure's hand boards by itself. Integrating smart objects into physical infrastructure can improve flexibility, reliability and efficiency in infrastructure operation. These benefits can reduce cost and manpower requirements as well as enhance safety. As trillions of things (objects) are connected to the Internet, it is necessary to have an adequate architecture that permits easy connectivity, control, communications, and useful applications. In form, with the change of functionality and structure design, the form will also change accordingly. Smart products must be equipped with intelligent form. As a result, various "wisdom" solutions have been emerged. For example, smart transportation, smart logistics, and smart healthcare systems are all based on the IoT at the core of technology. In the future, the realization of these "wisdom" solutions can solve many problems facing humanity's sustainable development such as shortage of resources, energy waste, and inefficiencies [7].

\subsection{Changes of design object}

The serviceability of design is the basic attribute of industrial design, which serves people's survival life relying on the design of various products. Industrial designers take charge of enhancing the user experience of a product by developing its outside shape and its user interface. The Internet has tremendously evolved in the last few years connecting billions of things globally. These things have different sizes, capabilities, processing and computational power and support different kind of applications. Therefore, with the development of science and technology, changes will be occurred in design requirements and design objects. In the IoT era the object of product design has changed from the original design of a lifeless product to the design of items with individualized intelligence and the networks behind the items. The products in traditional design involved human, machines, and the environment. In the era of IoT, they will become human, machines (under the network), environments, which increase the complexity of design. Because in the environment of network, designers will face a variety of needs since design is to serve the needs. There will be born new demands and new products every second, and in turn, a variety of new products will lead to the emergence of new requirements. New demands and new products are intertwined with each other to promote the upgrading of products constantly.

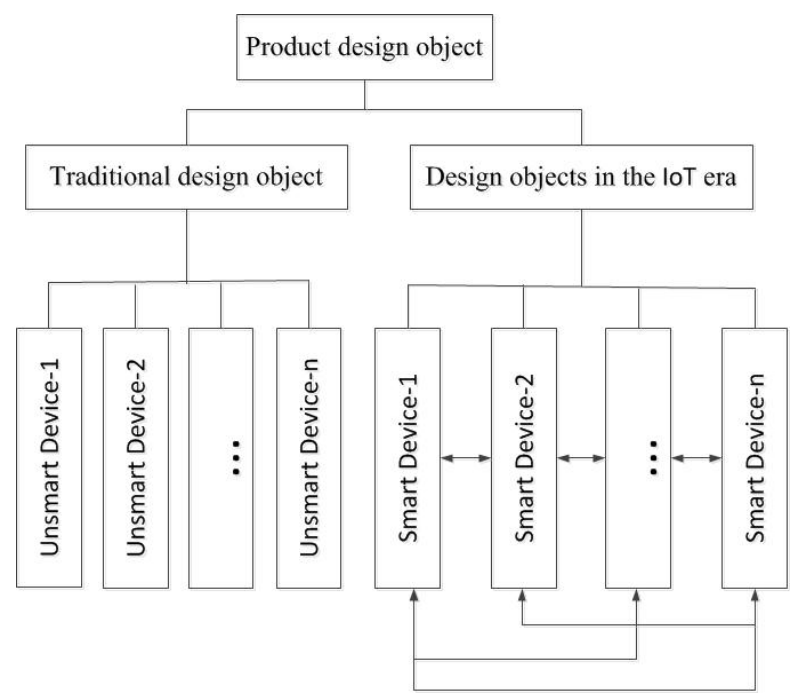

Figure 1. Compared with the traditional product design objects and IoT era design objects

Traditional product design objects must exchange information with the help of people. Where there is no information exchange between products. While in the IoT era, product design objects are devices with intelligence. With the advancement in technology, the devices processing power and storage capabilities significantly increased while their sizes reduced. These smart devices are usually equipped with different type of sensors and actuators. Large quantities of data were collected in the IoT environment. One future vision for the IoT is the Web of Things. The Web of Things proposes the use of web standards to fully integrate smart objects into the Worldwide Web. Using web technologies can make it easier for developers to build applications using smart 
objects and existing web protocols can more easily enable the interoperability and communication of different devices. Not only can these objects exchange information with people, but also can devices directly exchange information. As shown in Figure 1.

\subsection{Changes in the means to obtain design requirements}

Design requirements are the basis for creating excellent designs. Engineering and marketing models of product design and the product design process emphasize the importance of customer requirements. Ideally, companies that develop products ought to understand customer needs so that they can develop products that will sell. Assessments of customer needs are then used to specify functional requirements of the product. The functional requirements in turn are used to determine product design parameters. These three steps, from customer needs to functional requirements to design parameters are common for many established design methods including quality function deployment and axiomatic design. The product that has demand only has vitality. In the past, design requirements were obtained through observation, research, investigations, and interviews. In the IoT era, they can be obtained through the products themself. In the process of using the product (smart product), the user's demand for the product (smart product) will be reflected to the product (smart product). In the interaction between products (smart products), the demands between products (smart products) will also be generated. Through the IoT network, designers can obtain a variety of needs from users and smart products. In the IoT environment, all kinds of information can be fed back between smart products. Designers can obtain this information through certain means to analyze new potential needs and design more products that meet the needs of users. As shown in Figure 2.

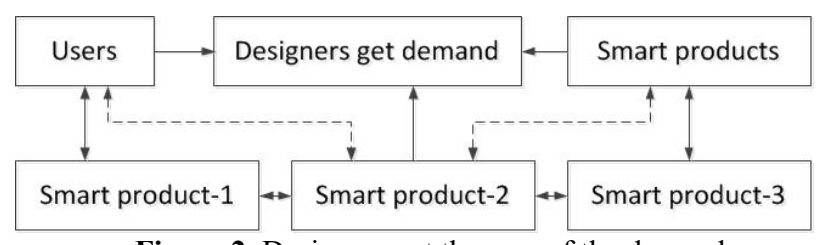

Figure 2. Designers get the way of the demand

\subsection{Changes in design procedures and methods}

Generally speaking, the design process mainly includes several stages, which are the previous market research and analysis, design and evaluation based on analysis, functional structure design, and production. The preliminary market survey analysis obtained problems during using product by face-to-face communication with users and potential users. While in the IoT era, it is only necessary to interact with existing products and enable realtime information exchange between products, which make design surveys more straightforward. Since this process more accurately reflects the user's applying at the time, the reliability of the information is high. In the IoT environment, a more objective assessment can be obtained and the assessment of design options and assessment methods can be carried out flexibly due to the scope of personnel involved in the assessment is very large and the smaller constraints on geographical and time.

\subsection{Changes in the design tool be applied}

Current design tools are limited to paper, pens, computers, etc. Future design tools will not be limited to these tools. It can also through various intelligent devices. These devices include personal computers, laptops, tablets, smart phones, PDAs and other hand-held embedded devices. The numbers of Internet connected devices are increasing at the rapid rate. These intelligent tools with intelligent information collection technology, sensor technology, sensing technology, thus can be used as industrial designers design tools. Under the IoT environment, human-machine interaction is no longer limited by time and place with the further development of technology, and it can realize remote-controlled computer aided industrial design, which can bring great convenience to designers. If our vision is correct, many IoT applications will be based on a deployed sensing, actuation, and communication platform (connecting a network of things). In these deployments, it is common for the devices to know their locations, have synchronized clocks, know their neighbor devices when cooperating, and have a coherent set of parameter settings such as consistent sleep/wake-up schedules, appropriate power levels for communication, and pair wise security keys.

\subsection{Change of design direction}

Since the IoT has not yet been realized, it might seem precocious to forecast the future directions of the IoT. However, future visions of the IoT will affect its current development and must therefore be considered. With the continuous development of IoT technology, industrial design direction will also undergo tremendous changes. Design on human-computer interaction will be the main design direction in the future. Given that IoT devices are likely to be connected to many objects and even to people themselves, examining the potential societal and personal impacts of the IoT is absolutely essential. Industrial design will play a more important role in the application of new technologies. In an IoT world, there exists a vast amount of raw data being continuously collected. It will be necessary to develop techniques that convert this raw data into usable knowledge. For example, in the medical area, raw streams of sensor values must be converted into semantically meaningful activities performed by or about a person such as eating, poor respiration, or exhibiting signs of depression. How to be safe and effective operation and avoiding misoperation, or even if the misoperation happened, but will not cause any damage, which need to be carefully studied for designers. Intelligent human-computer interaction will become an important design direction in the future. 


\section{Challenge for product designers}

How to satisfy the users' requirements to the greatest extent will be a great challenge for product designers in the future of the IoT. With the development of IoT technology, various people will have a variety of requirements. Some of these requirements are meaningful to designers and users, some are meaningless, how to filter, and how to translate these requirements into products need the wisdom of designers. Industrial designers must have such ability and take on this responsibility.

The products and features of the IoT are mainly reflected in the following: 1) the frequency of information interaction is greatly increased. The previous information exchange needs to be carried out by people. In the IoT environment, products and products can be directly carried out, greatly reducing the burden on people, and to a certain extent, the transition from liberating physical strength to emancipating the mind. 2) The interaction between products and people is no longer limited by the spatial distance. 3) The product has certain wisdom. With a variety of advanced technologies, users can effectively use and manage products. At the same time, the ubiquity and interactions involved in IoT will provide many conveniences and useful services for individuals, but also create many opportunities to violate privacy. To solve the privacy problem created by IoT applications of the future, the privacy policies for each (system) domain must be specified. Once specified, either the individual IoT application or the IoT infrastructure (e.g., the utility capability) must enforce privacy. Consequently, the IoT paradigm must be able to express users' requests for data access and the policies, such that the requests can be evaluated against the policies in order to decide if they should be granted or denied. A new language is required to express privacy policies because the requirements not easily expressed in current privacy languages. It is needed to represent different types of data owners and request subjects in the system as well as external users and their rights when domains interact.

\section{Conclusions}

The IoT era will be the age of information explosion; the interaction of information will be frequent between products, and between people and products. By bringing existing technologies together in a novel way, the IoT has the potential to reshape our world. The IoT embeds intelligence in the sensor devices to autonomously communicate, exchange information and take intelligent decisions. Simply, IoT transitions human-human communication to human-human, human-device and devicedevice communication. The IoT holds the promise of improving people's lives through both automation and augmentation. The capabilities offered by the IoT can save people and organizations time and money as well as help improve decision making and outcomes in a wide range of application areas. In this context, "design" will be more reflected in "integrity" and "integration" aspects. Industrial designers need to grasp the frontiers of design, focus on exploring ways of innovation in the "goods, processes, and services", pay more attention and research on the IoT, and apply the IoT technology, using various design methods, and thus better service to the user, better to provide higher quality design services for this era. This article put forward some suggestions on issues in the era of IoT that industrial designers should pay attention. It elaborates on the aspects of design content, design objects, design procedures and methods, means of obtaining design requirements, design tools, and changes in design direction. Subsequent research will focus on the interaction between humans and smart products in the IoT environment and the interaction between smart products.

\section{Acknowledgment}

This work was supported by National Key Technology R\&D Program, China (Grant No. 2015BAH21F01) and the 111 Project, (Grant No.B13044).

\section{References}

1. S. Nath, S. Som, Indian J. Sci. Technol., 10 (3), 1-5 (2017)

2. T. Kramp, R. van Kranenburg, S. Lange, Introduction to the internet of things (2013)

3. C. W. Tsai, C. F. Lai, M. C. Chiang, L. T. Yang, IEEE Commun. Surv. Tutorials, 16 (1), 77-97 (2014).

4. S.Balasubramaniam, J. Kangasharju, Computer (Long. Beach. Calif)., 46(2), 62-68 (2013)

5. T. Jenkins, I. Bogost, the 32nd annual ACM conference on Human factors in computing systems - CHI EA'14, 731-740 (2014)

6. A. Whitmore, A. Agarwal, and L. Da Xu, Inf. Syst. Front., 17 (2), 261-274 (2015)

7. F. Khodadadi, A. V. Dastjerdi, R. Buyya, Internet of Things: Principles and Paradigms, 3-27 (2016)

8. S. Li, L. Da Xu, S. Zhao, Inf. Syst. Front., 17 (2) 243-259 (2015)

9. Y. Yu, J. Wang, G. Zhou, ICDLE 2010 - 2010 4th International Conference on Distance Learning and Education, Proceedings, 74-77 (2010)

10. J. A. Stankovic, IEEE Internet Things J., 1 (1), 3-9 (2014)

11. A. Al-Fuqaha, M. Guizani, M. Mohammadi, M. Aledhari, M. Ayyash, IEEE Commun. Surv. Tutorials, 17 (4) 2347-2376 (2015)

12. Y.-K. Chen, 17th Asia and South Pacific Design Automation Conference,383-388 (2012)

13. R. Khan, S. U. Khan, R. Zaheer, S. Khan, 10th International Conference on Frontiers of Information Technology, FIT 2012, 257-260 (2012) 